\title{
Acoustic Property Measurements in a Photoacoustic Imager
}

\author{
René G. H. Willemink ${ }^{a}$, Srirang Manohar ${ }^{b}$, Cornelis H. Slump ${ }^{a}$, Ferdi van der Heijden ${ }^{a}$ and \\ Ton van Leeuwen ${ }^{b}$ \\ ${ }^{a}$ Institute for Biomedical Technology (BMTI), Signals and Systems Group (SaS), Faculty of \\ Electrical Engineering (EWI), University of Twente, Postbox 217, 7500AE Enschede, The \\ Netherlands; \\ ${ }^{b}$ Institute for Biomedical Technology (BMTI), Biophysical Engineering Group, Faculty of \\ Applied Physics (TNW), University of Twente, Postbox 217, 7500AE Enschede, The \\ Netherlands
}

\begin{abstract}
Photoacoustics is a hybrid imaging technique that combines the contrast available to optical imaging with the resolution that is possessed by ultrasound imaging. The technique is based on generating ultrasound from absorbing structures in tissue using pulsed light. In photoacoustic (PA) computerized tomography (CT) imaging, reconstruction of the optical absorption in a subject, is performed for example by filtered backprojection. The backprojection is performed along circular paths in image space instead of along straight lines as in X-ray CT imaging. To achieve this, the speed-of-sound through the subject is usually assumed constant. An unsuitable speed-of-sound can degrade resolution and contrast. We discuss here a method of actually measuring the speedof-sound distribution using ultrasound transmission through the subject under photoacoustic investigation. This is achieved in a simple approach that does not require any additional ultrasound transmitter. The method uses a passive element (carbon fiber) that is placed in the imager in the path of the illumination which generates ultrasound by the photoacoustic effect and behaves as an ultrasound source. Measuring the time-of-flight of this ultrasound transient by the same detector used for conventional photoacoustics, allows a speed-of-sound image to be reconstructed. This concept is validated on phantoms.
\end{abstract}

Keywords: photoacoustic imaging, ultrasound, speed of sound, tomography

\section{INTRODUCTION}

Photoacoustic (PA) imaging is a new noninvasive medical imaging modality. It is a technique which is believed to be harmless for the human body ${ }^{1}$ and uses pulsed optical or microwave energy. The process is based on the absorption of the pulse of input energy by an object, resulting in spatial distribution of temperature increases. This temperature distribution leads to the generation of pressure waves, ${ }^{2}$ which can be measured outside of the object. An image of the optical absorption distribution can be reconstructed from the measured acoustic signals at the boundary of the object. ${ }^{3}$

The measured acoustic signals are not only dependent on the optical absorption distribution, but also on the acoustic property distribution of the object. An often used assumption in PA imaging is that the acoustic properties of the imaged object are homogeneous. However, this assumption is violated in many objects, for instance objects consisting of different types of soft tissue, where each type of soft tissue can have a slightly different speed of sound or acoustic attenuation factor. The result of, for example, assuming a constant value for the speed of sound in an object actually having an inhomogeneous speed of sound will be that the measurement model describing the relation between optical absorption and observed acoustic signals is incorrect. Reconstructing an image of the optical absorption distribution requires inversion of this measurement model and consequently results in an incorrect reconstruction of the optical absorption distribution. When the distribution of the acoustic property of the object is known beforehand it can be incorporated into the measurement model. Using the improved measurement model in the reconstruction phase will result in better agreement of the reconstructed optical absorption distribution with the actual optical absorption distribution.

Author for correspondence: rene.willemink@utwente.nl 
Several authors have considered the case of incorporating acoustic property inhomogeneities into the PA imaging problem. $\mathrm{Xu}$ and $\mathrm{Wang}^{4}$ studied the effects of acoustic heterogeneities in PA breast imaging. The acoustic property distribution was modeled with a speed of sound map consisting of a boundary velocity and an inner part with a different velocity. Based on the known speed of sound map, the measurement relation between the optical absorption distribution and obtained acoustic measurements at the boundary was inverted to obtain the optical absorption distribution. The work was later extended ${ }^{5}$ by allowing arbitrary speed of sound distributions and using Ultrasound Transmission Tomography to recover the speed of sound distribution first.

Jiang et $\mathrm{al}^{6}$ also incorporated acoustic property inhomogeneities into the problem. In this case both optical and acoustic properties were obtained from only PA measurements. The measurements are evaluated in a wave equation by relating both the acoustic property distribution and the optical property distribution to observed acoustic measurements. A finite element method was used to discretize the wave equation. The discretized system forms a set of non-linear equations relating acoustic and optical properties to the observed acoustic measurements. The acoustic and optical property distributions are then recovered by an iterative Newton method combined with Marquardt and Tikhonov regularizations.

La Rivière ${ }^{7}$ studied the effects of acoustic dispersion and attenuation (two dependent parameters) on PA imaging. In this case there was no acoustic property distribution reconstructed. The acoustic property was assumed constant over the object, but the effects of dispersion and attenuation were investigated. A relation between observed PA signals and dispersion free PA signals based on a known attenuation constant was formed. Inversion of this relation gives the dispersion free PA signals which can be used in the reconstruction process to obtain an improved optical absorption distribution reconstruction.

\section{APPROACH}

In our method we can obtain both optical and acoustic property distributions of the object in only one measurement and by using only one form of input energy. ${ }^{8}$ We make use of the photoacoustic effect to transform the optical input energy into acoustic energy. The acoustic signal originating from the object is dependent on both its optical and its acoustic properties. By adding an extra, carefully positioned, passive element to our setup that converts a part of the incident optical energy into acoustic energy in the form of a short acoustic pulse, we can obtain isolated measurements of the object's acoustic properties. The passive element will be placed at the opposite side of the object with respect to the ultrasound sensor array. Consequently the generated acoustic pulse will have to travel through the object in order to arrive at the ultrasound sensor array. The acoustic property distribution inside the object will influence the output signal of the passive element that is received with the ultrasound sensor. Thus the measured signal of the generated acoustic pulse contains information about acoustic properties of the object travelled by the acoustic pulse.

In the following part of this section we will first illustrate the experimental setup. Then we show in several steps how this setup can be used to reconstruct a speed of sound (SOS) map of the imaged object. In this work we assume that the acoustic waves travel along rectilinear paths, meaning we will ignore refraction effects. This simplification allows us to reconstruct a speed of sound map based on time of flight values obtained from a set of known source-detector pair measurements. The final goal in this work is to obtain the speed of sound map, integrating the resulting speed of sound map in PA image reconstruction will be considered in future work.

\subsection{Experimental setup}

Our experimental setup is schematically displayed in Figure 1. The object is mounted on a rotary stage and is immersed in an imaging tank filled with water. An illuminating fiber bundle is located at one side of the tank and at the opposite side an ultrasound (US) sensor array is placed. The passive element is positioned just in front of the illuminating fiber bundle. As passive element a carbon fiber was used. When the laser fires a short pulse of light, both the object and the passive element will generate acoustic signals as depicted, due to the photoacoustic effect. The object and passive element are positioned in such a way with respect to the US sensor array, that the two generated signals which are received by the sensor are separated in time.

During measurement acquisition the object is rotated stepwise over a range of $360^{\circ}$. At each step the laser fires a pulse of light and ultrasound signals are recorded with the sensor array. 


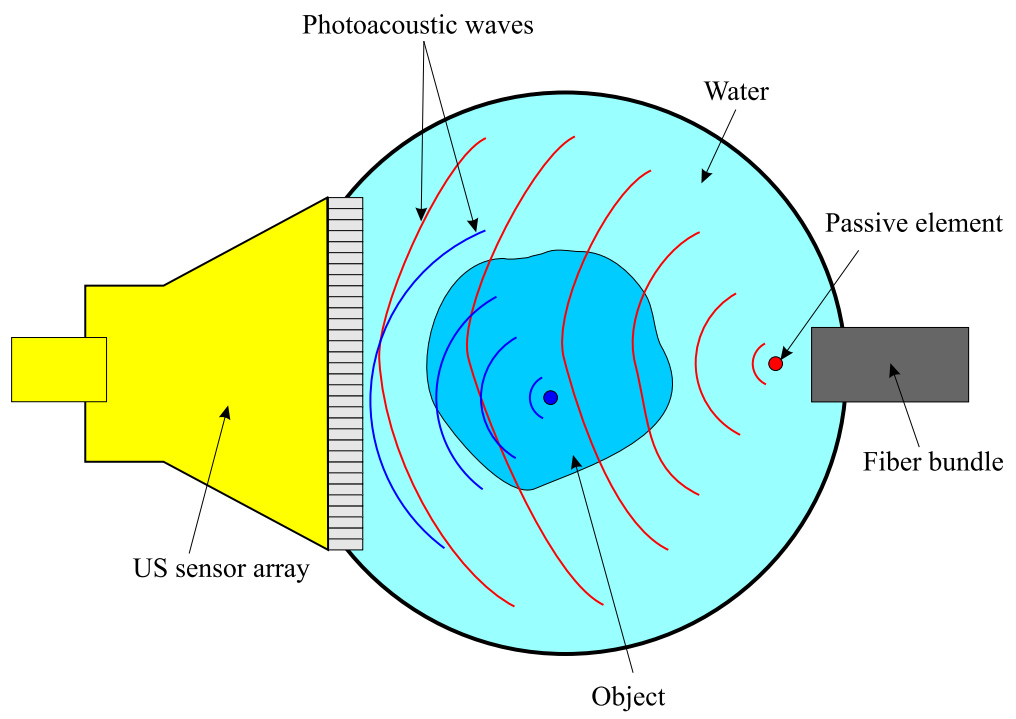

Figure 1: Experimental setup

\subsection{Time of flight sinogram extraction}

The first step in our method is the extraction of a time of flight sinogram from the measurements. This time of flight sinogram contains the time of flight of the acoustic pulse generated by the passive element, indexed for each sensor element number of the array and for each rotation angle at which the time of flight was measured. To extract a time of flight value from a signal trace, we cross correlate a template signal of the passive element with a region of interest of the signal trace. By doing so, we assume that dispersion between source and detector can be ignored so that the signal shape (up to a scale factor) is independent on the travelled path. The boundaries of the region of interest are obtained by calculating expected lowest and highest time of flight values based on prior expected speed of sound variations inside the object. By considering only this region of interest, we have no interference with PA signals generated by the object.

\subsubsection{Reference (calibration) measurement}

Before a session is started, a reference measurement is always made. This reference measurement serves the purpose of calibrating the position of the passive element as well as estimating the speed of sound of the surrounding medium. The reference measurement is obtained by removing the object from the imaging tank. A measurement is then obtained with only PA signals from the passive element passing only through the background medium.

The first step is the estimation of the passive element signal template, which can be used later in the cross correlation phase. When a dispersionless background medium is used, such as water ${ }^{9}$ the template signal generated by the passive element can be estimated from the time domain signal traces. Our approach here is to use the signal obtained from the first sensor element as an initial estimate of the template and cross correlate the signals obtained from all other sensors to this initial estimate. The other signals are then shifted in time according to their maximum cross correlation. Averaging over all signals gives then an improved estimate of the template where the noise variance is reduced by a factor corresponding to the number of sensor elements used in the estimate. This procedure is then repeated again, where the improved estimate is used as initial estimate and cross correlation is performed against this improved estimate. The resulting average contains the actual template with an arbitrary starting and ending point. By looking at the envelope of the obtained template, the starting point and ending point of the template are found by thresholding the envelope of the signal. The intermediate steps and results of this procedure are displayed in Figure 2.

The obtained delay values by cross correlation with the template signal are subsequently used for the calibration of several parameters for the current session. These parameters include the exact position of the passive 


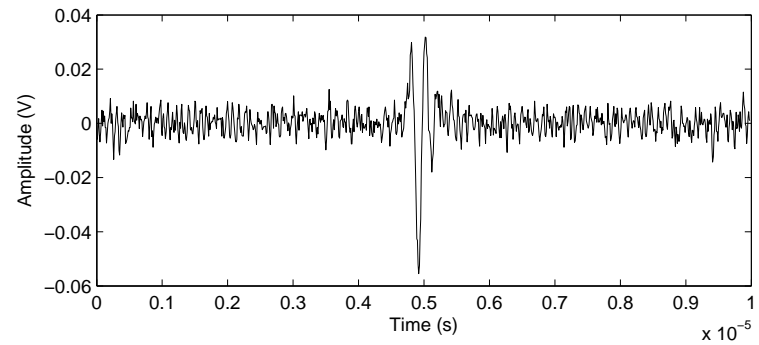

(a) Reference signal from first sensor element

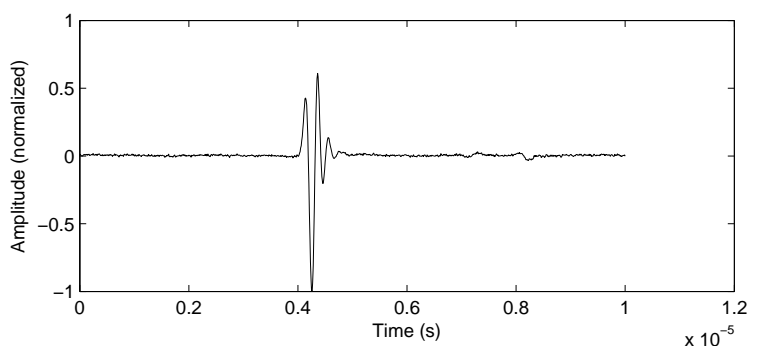

(c) Averaged signal over all sensor elements

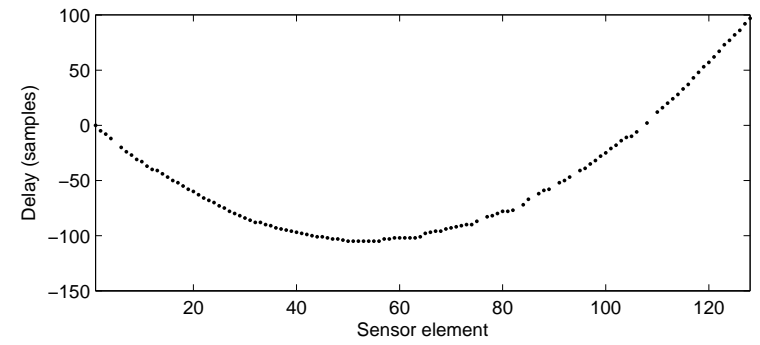

(b) Delays obtained by cross correlation with the signal from the first sensor element

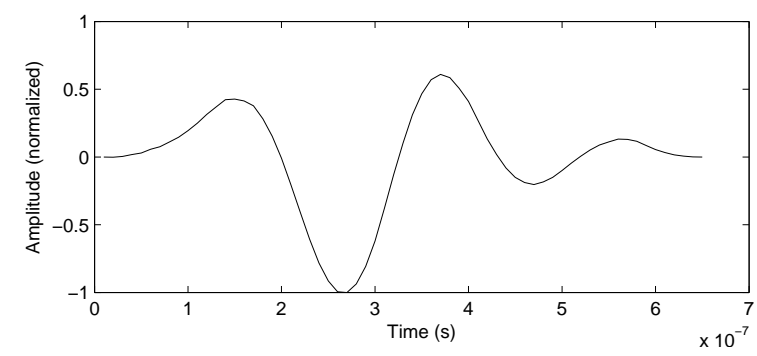

(d) Template signal of the passive element

Figure 2: Estimation of the template signal

element and the speed of sound of the background medium. To calibrate these parameters a measurement model based on the geometry of the setup was used. This model defines the relation between the parameters and the measured time of flight values from the passive element to each of the sensor elements. The parameter values are estimated by minimizing the least squares error between observed and predicted measurements based on the model.

\subsubsection{Generating the sinogram}

The sinogram of the time of flight values for the measurement with the object inside the imaging tank is obtained by calculating the time of flight value for each sensor element at each angle separately. This is done in two steps. In the first step a cross correlation of the passive element signal template with the measured signal trace is calculated, and the time of flight value at which the cross correlation is maximal is used as an initial time of flight value. This first coarse cross correlation is performed around integer samples. In the second setup we go to sub sample accuracy by maximizing the cross correlation based on interpolation via the frequency domain. We make use of the identity that shifting in the time domain amounts to a multiplication in the frequency domain:

$$
y(t-\tau)=\mathcal{F}^{-1}\left\{\mathcal{F}\{y(t)\} \cdot e^{-j \omega \tau}\right\}
$$

Here $\mathcal{F}$ indicates the Fourier transform and $\mathcal{F}^{-1}$ the inverse Fourier transform. Using this relation we can calculate cross correlations at sub sample accuracy by shifting in the frequency domain using FFT transforms. We have chosen a sub sample resolution of ten times the original sampling period. We then calculate cross correlations over one sampling period interval in ten steps around the initially obtained maximum.

\subsection{SOS map reconstruction}

The sinogram contains time of flight values indexed for each sensor element number and each projection angle. The SOS distribution is recovered in a 2-dimensional plane (a slice of the 3-dimensional structure) spanned by the US sensor array and the position of the passive element. Each time of flight value is related to the SOS distribution according to:

$$
t o f_{n, \phi}=\int_{l_{n, \phi}} \frac{1}{c(\boldsymbol{r})} \mathrm{d} \boldsymbol{r}
$$


where $n$ is the sensor element number, $\phi$ the projection angle, $l_{n, \phi}$ the acoustic path between source and detector for a given $n$ and $\phi, c(\boldsymbol{r})$ is the speed of sound distribution and $\boldsymbol{r}$ is the integration variable representing position. Since we ignore diffraction effects in this study, the path $l_{n, \phi}$ is rectilinear. This means the paths in the reference measurement (with only background medium) and the object measurements coincide. Time of flight delays with respect to the reference measurement can thus be calculated, which is more suitable for the pertinent inverse problem. Time of flight delays are related to the speed of sound distribution as:

$$
t_{n, \phi}=\int_{l_{n, \phi}}\left(\frac{1}{c(\boldsymbol{r})}-\frac{1}{c_{0}}\right) \mathrm{d} \boldsymbol{r}=\int_{l_{n, \phi}} \tau(\boldsymbol{r}) \mathrm{d} \boldsymbol{r}
$$

where $t_{n, \phi}$ is the time of flight delay, $c_{0}$ is the speed of sound of the background medium and $\tau(\boldsymbol{r})$ is the delay per unit distance distribution. We are interested in reconstructing a sampled representation of $\tau(\boldsymbol{r})$ on a set of grid points. The distribution $\tau(\boldsymbol{r})$ will be constructed from the sampled representation by bilinear interpolation. To do so we use a set of $N$ triangular basis functions ${ }^{10} h_{i}(\boldsymbol{r})$ covering our domain of interest (the object):

$$
\tau(\boldsymbol{r})=\sum_{i=1}^{N} x_{i} h_{i}(\boldsymbol{r})
$$

where $\left\{x_{i}\right\}_{i=1}^{N}$ is the sampled representation of $\tau(\boldsymbol{r})$. We subsequently calculate the integral in (3) by sampling along the acoustic path $l_{n, \phi}$ at individual locations separated by a step size equal to the resolution of the grid. This results in linear equations relating the observed time of flight delays to the unknown delay per unit values:

$$
t_{n, \phi}=\boldsymbol{h}_{n, \phi}^{T} \boldsymbol{x}
$$

with $\boldsymbol{h}_{n, \phi}$ a vector that calculates the sampled integral for sensor element $n$ at angle $\phi$ when operated on $\boldsymbol{x}$ and $\boldsymbol{x}=\left[x_{1}, \ldots, x_{N}\right]^{T}$ is a vector containing the delay per unit values. By combining all sensor elements at all angles a linear system of equations is formed:

$$
\boldsymbol{t}=H \boldsymbol{x}
$$

where $\boldsymbol{t}$ and $H$ are a vector and matrix consisting of the time of flight delays $t_{n, \phi}$ and the vectors $\boldsymbol{h}_{n, \phi}^{T}$ for all sensor elements and all angles. The delay per unit distance distribution can now be calculated by solving for $\boldsymbol{x}$ in (6).

Because inverting Eq. (6) is quite sensitive to noise in $\boldsymbol{t}$ we assume that there is a degree of smoothness on the solution. The amount of smoothness depends on the prior information of the imaged object and can be specified by a probability density function of the value of the gradient of the expected image. The relation in (6) can also be specified by a probability density function (pdf) representing the likelihood of the observed measurements based on the noise characteristics of measuring $\boldsymbol{t}$. The optimal value for $\boldsymbol{x}$ can then be obtained by maximizing the product of these two pdf's.

$$
\boldsymbol{x}=\underset{\boldsymbol{x}}{\operatorname{argmax}} p_{H}(\boldsymbol{t} \mid \boldsymbol{x}) p_{G}(\boldsymbol{x})
$$

where $p_{H}(\boldsymbol{t} \mid \boldsymbol{x})$ represents the likelihood of $\boldsymbol{x}$ given the measurements $\boldsymbol{t}$ using relation (6) and $p_{G}(\boldsymbol{x})$ represents the prior distribution of the gradient of $\boldsymbol{x}$. In the case of additive Gaussian white noise in $\boldsymbol{t}$ with variance $\sigma_{\boldsymbol{t}}^{2}$ and additive Gaussian white noise on the gradient with variance $\sigma_{G}^{2}$, the maximum can be found by solving:

$$
\boldsymbol{x}=\underset{\boldsymbol{x}}{\operatorname{argmin}}\left\{\|\boldsymbol{t}-H \boldsymbol{x}\|^{2}+\|\lambda G \boldsymbol{x}\|^{2}\right\}=\underset{\boldsymbol{x}}{\operatorname{argmin}}\left\|\left[\begin{array}{c}
\boldsymbol{t} \\
\mathbf{0}
\end{array}\right]-\left[\begin{array}{c}
H \\
\lambda G
\end{array}\right] \boldsymbol{x}\right\|^{2}
$$

where $\lambda=\frac{\sigma_{t}}{\sigma_{G}}$ and $G$ is the gradient operator. Because both matrices $H$ and $G$ are large and also sparse, we can efficiently use the iterative LSQR algorithm ${ }^{11}$ to find a solution to Eq. (8). The procedure will be stopped after a limited number of iterations (around 10) due to the semi-convergence property of this iterative method.

Finally we get a speed of sound distribution from the obtained time delay per unit distance distribution by sampling $\tau(\boldsymbol{r})$ at the center points of $h_{i}(\boldsymbol{x})$ and again using bilinear interpolation for the off-grid points to represent $c(\boldsymbol{r})$ :

$$
c(\boldsymbol{r})=\sum_{i=1}^{N} \frac{h_{i}(\boldsymbol{r})}{x_{i}+\frac{1}{c_{0}}}
$$




\section{EXPERIMENTAL RESULTS}

Experiments on several phantom objects were performed to verify our approach. We will discuss experiments on four different objects, a short description of each of the experiments is given in Table 1. The surrounding medium was distilled water $(\sim 1495 \mathrm{~m} / \mathrm{s})$ for all of the four experiments. Experiment 1 and experiment 3 share the same phantom object, however their position inside the imaging tank was different. The phantom used in experiment 1 was placed further away from the sensor array and the phantom used in experiment 3 was placed closer to the US sensor array. The positions of the phantoms used in experiment 2 and 4 were approximately the same as in experiment 3.

Table 1: Overview of the experiments

\begin{tabular}{|l|l|}
\hline Nr. & Description \\
\hline 1 & Agar phantom $(\sim 1500 \mathrm{~m} / \mathrm{s})$, cylindrical cavity filled with olive oil $(\sim 1465 \mathrm{~m} / \mathrm{s})$ \\
2 & Agar phantom $(\sim 1500 \mathrm{~m} / \mathrm{s})$, two square shaped cavities filled with olive oil $(\sim 1465 \mathrm{~m} / \mathrm{s})$ \\
3 & Agar phantom $(\sim 1500 \mathrm{~m} / \mathrm{s})$, cylindrical cavity filled with olive oil $(\sim 1465 \mathrm{~m} / \mathrm{s})$ \\
4 & One half Agar $2 \%(\sim 1503 \mathrm{~m} / \mathrm{s})$, one half Agar $4 \%(\sim 1507 \mathrm{~m} / \mathrm{s})$ \\
\hline
\end{tabular}

\subsection{Calibration}

Before a speed of sound map can be reconstructed from obtained measurements, it is important to know the geometrical parameters of the setup accurately. To ensure this we calibrated each experiment. For the calibration procedure, the phantom object was removed from the setup after SOS measurements were taken and subsequently a reference measurement was made. A geometrical model was fitted to the reference measurement and the parameters found are then used in the speed of sound map reconstruction phase. The estimated geometrical parameters after calibration are displayed in Table 2 and the corresponding fit to the reference measurements is displayed in Figure 3.

\begin{tabular}{|c|r|r|r|}
\hline Nr. & $c_{0}(\mathbf{m} / \mathbf{s})$ & $p_{x}(\mathbf{m m})$ & $p_{y}(\mathbf{m m})$ \\
\hline 1 & 1499 & 89.0 & -1.0 \\
2 & 1502 & 89.2 & -0.7 \\
3 & 1500 & 89.1 & -0.8 \\
4 & 1493 & 88.7 & 3.3 \\
\hline
\end{tabular}

Table 2: Calibration results on several phantoms, $c_{0}$ is the speed of sound of the surrounding medium, $p_{x}$ the distance of the passive element to the US sensor array and $p_{y}$ the positioning offset from the center of the US sensor array

\subsection{Extraction of the sinogram}

A sinogram was extracted for each experiment as described in Section 2.2.2. In Figure 4a the extracted sinogram for experiment 1 is displayed. We have noticed that the obtained sinograms contains a discontinuity when going from the last projection angle to the first projection angle. This discontinuity is not what should be expected, rotating the setup by only a small amount should just give a slightly different projection. The sinograms seem to have a drifting offset with increasing projection angle. Our physical explanation for the drifting offset is heating of the imaging tank during measurements due to the laser illuminating the setup. Because of this heating, the speed of sound in the surrounding medium is increased, leading to a shorter time of flight. In the following section were we show the results of reconstruction of the speed of sound maps, we correct the sinograms for the temperature heating effect by removing the drifting offset as shown in Figure 4b.

\subsection{Speed of sound map reconstruction}

Based on the estimated geometry parameters and extracted sinograms, speed of sound maps were reconstructed for each experiment as described in Section 2.3. The resulting images are displayed in Figure 5.

The reconstructions look very promising and correctly show the structure of each of the four phantoms. Also the reconstructed speed of sound values are in good agreement with the true speed of sound inside the used 


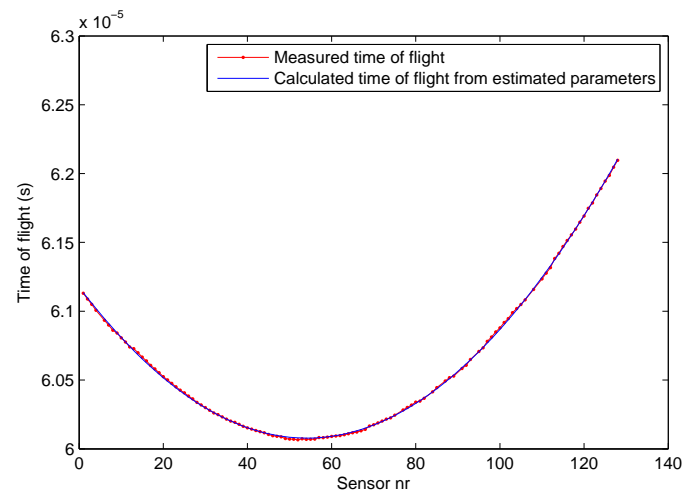

(a) Experiment 1

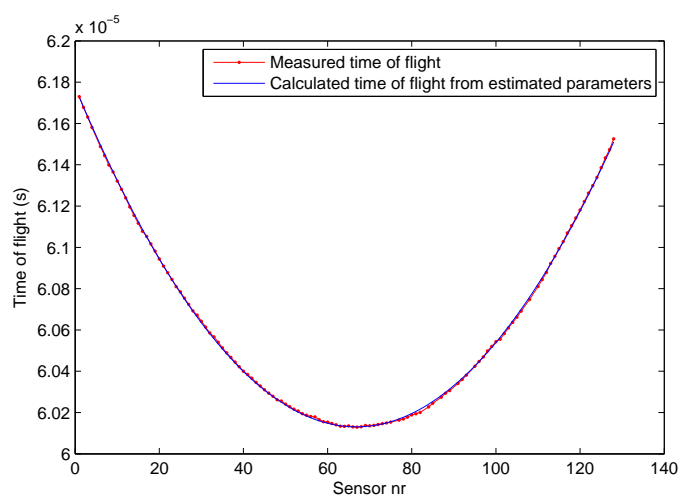

(c) Experiment 3

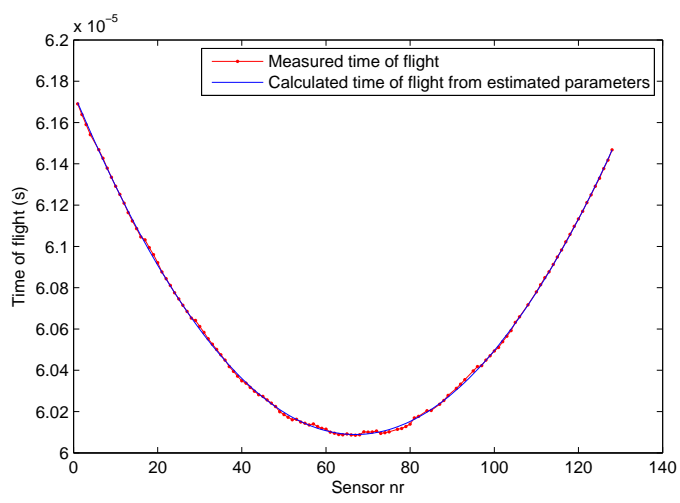

(b) Experiment 2

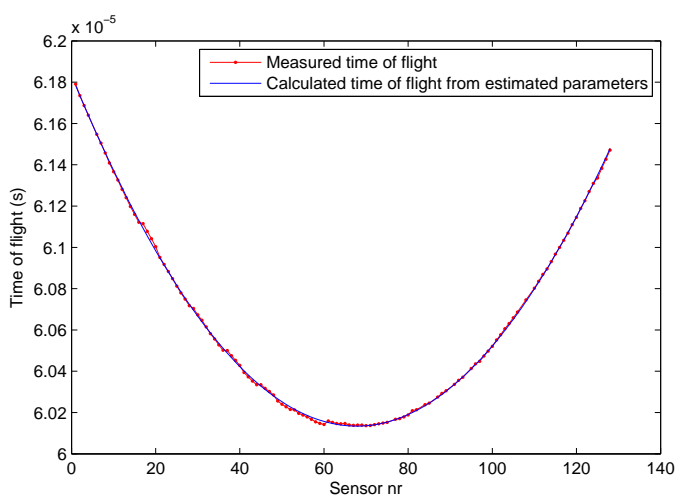

(d) Experiment 4

Figure 3: Obtained reference measurements and predicted reference measurements after calibration of the geometry model parameters

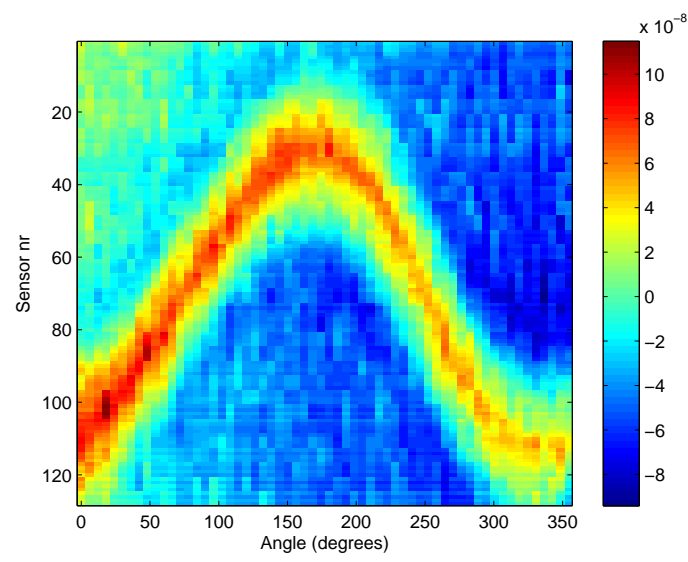

(a) Extracted time of flight delay sinogram

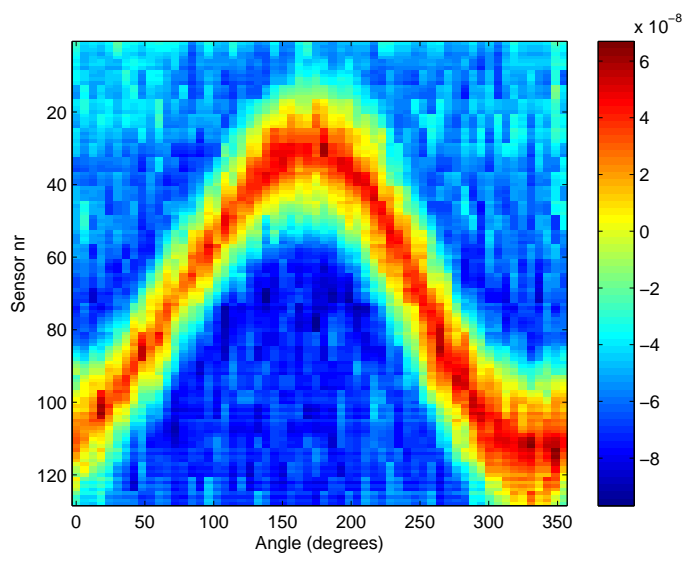

(b) Temperature drift corrected time of flight delay sinogram

Figure 4: Sinogram obtained in experiment 1 


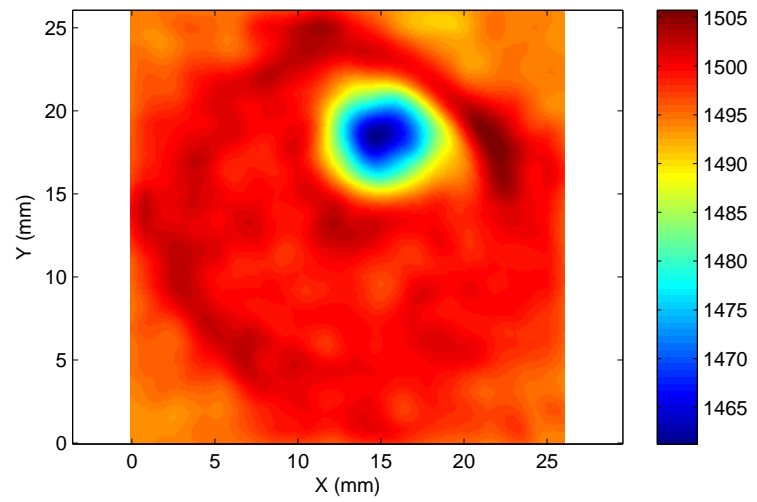

(a) Experiment 1

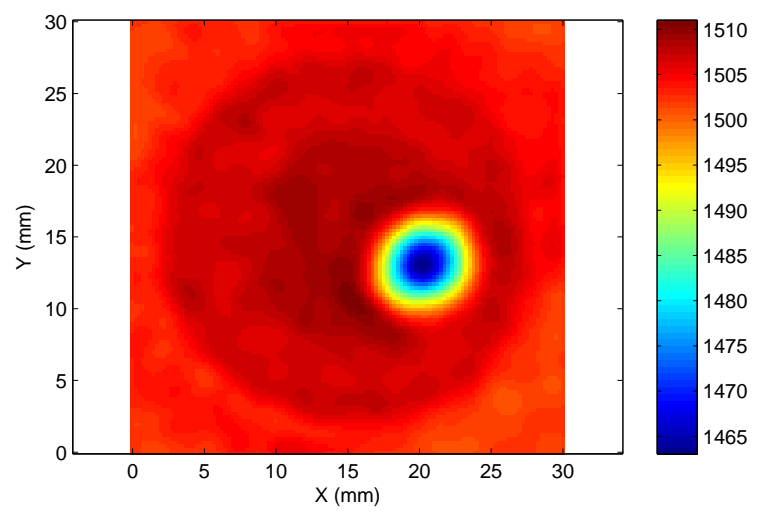

(c) Experiment 3

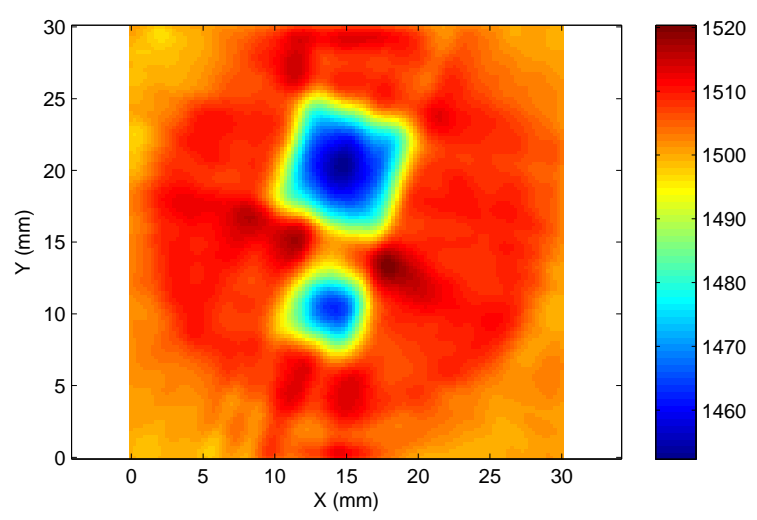

(b) Experiment 2

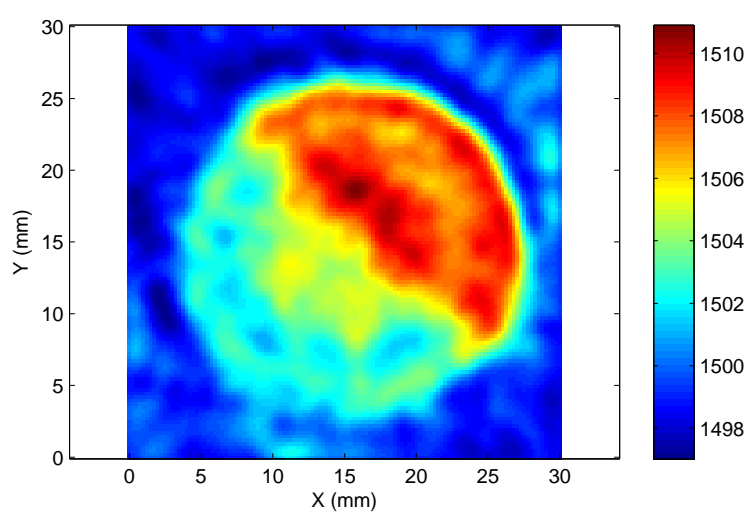

(d) Experiment 4

Figure 5: Speed of sound map reconstruction results for each of the used phantom objects. The reconstructed speed of sound distribution is displayed in color where the colorbar next to each image shows the corresponding value in $\mathrm{m} / \mathrm{s}$

phantoms. The reconstruction accuracy of the phantom used in both experiment 1 and 3 is clearly better in experiment 3. This is due to the fact that in experiment 1, the phantom was placed too far away from the US sensor array so that there is no clear contrast visible between surrounding water and the phantom. Care should thus be taken that the phantom is fully contained inside the fanbeam shape defined by the US sensor array and the passive element.

Experiment number 4 shows that low contrast imaging is possible. Speed of sound differences being as low as $3 \mathrm{~m} / \mathrm{s}$ are very well visible in the reconstructed images.

\section{CONCLUSION}

We have shown that our proposed technique allows us to reconstruct speed of sound distributions from photoacoustic measurements by adding a passive element to our setup. The reconstruction results closely resemble the true structure of the phantoms. The phantoms that were used are comparable to real soft tissue in the sense 
that the distribution of speed of sound values corresponds to expected values of real soft tissue. In this study diffraction effects, i.e. the bending of rays, were not taken into account. This will be considered in follow up studies to see how much improvement in image quality can be gained.

The phantoms considered here did not yet contain any optical absorption. We will however continue our study by including optical absorbers into the phantom as well.

\section{REFERENCES}

1. M. Xu and L. V. Wang, "Photoacoustic imaging in biomedicine," Rev. of Sci. Instrum. 77(4), 2006.

2. M. W. Sigrist and F. K. Kneubhl, "Laser-generated stress waves in liquids," J. Acoust. Soc. Am. 64, pp. 1652-1663, 1978.

3. R. A. Kruger, P. Liu, Y. R. Fang, and C. R. Appledorn, "Photoacoustic ultrasound (paus)-reconstruction tomography," Med. Phys. 22(10), pp. 1605-1609, 1995. 0094-2405.

4. Y. Xu and L. V. Wang, "Effects of acoustic heterogeneity in breast thermoacoustic tomography," Phys. in Med. and Biol. 51, pp. 6437-6448, 2003.

5. X. Jin and L. V. Wang, "Thermoacoustic tomography with correction for acoustic speed variations," IEEE Trans. on Ultr., Ferr. and Freq. Contr. 50(9), pp. 1134-1146, 2003.

6. H. Jiang, Z. Yuan, and X. Gu, "Spatially varying optical and acoustic property reconstruction using finiteelement-based photoacoustic tomography," J. Opt. Soc. Am. A 23, pp. 878-888, April 2006.

7. P. J. L. Rivière, "Image reconstruction in optoacoustic tomography for dispersive acoustic media," $O p t$. Let. 31(6), pp. 781-783, 2006.

8. S. Manohar, R. G. H. Willemink, F. van der Heijden, C. H. Slump, and T. G. van Leeuwen, "Concomitant speed-of-sound tomography in a photoacoustic imager," Submitted to Appl. Phys. Lett. , 2007.

9. C. W. Horton, "Dispersion relationships in sediments and sea water," J. Acoust. Soc. Am. 55(3), pp. 547$549,1974$.

10. K. M. Hanson and G. W. Wecksung, "Local basis-function approach to computed tomography," Appl. Opt. 24(23), pp. 4028-4039, 1985.

11. C. C. Paige and M. A. Saunders, "LSQR: An algorithm for sparse linear equations and sparse least squares," ACM Trans. on Math. Softw. 8(1), pp. 43-71, 1982. 